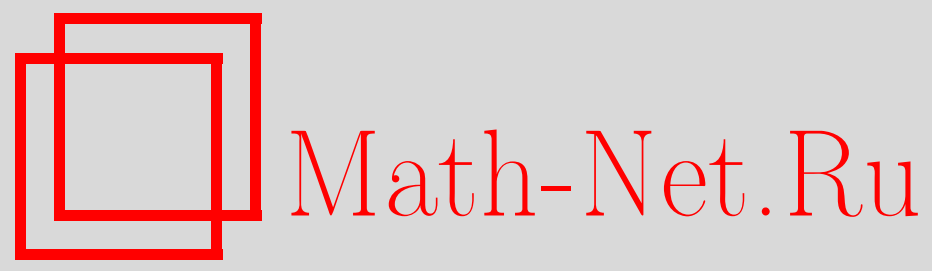

М. А. Сидоров, Использование модели скрытого марковского поля для стегоанализа изображений, Дискрет. матем., 2005, том 17, выпуск 3, 89-104

DOI: https://doi.org/10.4213/dm118

Использование Общероссийского математического портала Math-Net.Ru подразумевает, что вы прочитали и согласны с пользовательским соглашением http: //www . mathnet.ru/rus/agreement

Параметры загрузки:

IP : 3.89 .197 .203

26 апреля 2023 г., 13:51:22

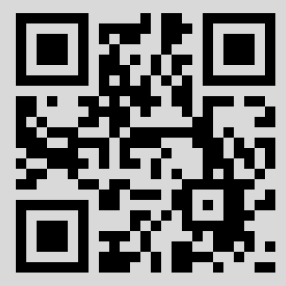




\title{
Использование модели скрытого марковского поля для стегоанализа изображений
}

\author{
() 2005 г. М. А. Сидоров
}

\begin{abstract}
Рассматривается метод решения задачи стегоанализа с использованием аппарата скрытых марковских полей в случае LSB-модификации исходного дискретного марковского поля. Получены итерационные алгоритмы фильтрации, позволяющие оценивать параметры скрытого марковского поля с заданными свойствами.
\end{abstract}

\section{1. Введение}

Задачи по выделению шумовой составляющей различной природы из цифровых изображений периодически возникают в различных областях современной жизни, таких как медицина, астрономия и телевидение. В настоящее время среди специалистов в области обработки изображений возрос интерес к методам на основе дискретных марковских полей [1] и, в частности, к методам, использующим скрытые марковские модели (СММ) [2]. Последние интересны тем, что позволяют весьма качественно определить параметры как исходного изображения так и шума, при анализе только зашумленного изображения. Первоначально их эффективность была оценена при решении задач распознавания, в частности, распознавания речи [3].

В работах $[4,5]$ было предложено использовать теорию СММ для решения задач стегоанализа, которые состоят в выявлении скрытой передачи информации, встраиваемой во всевозможные (мультимедиа-) контейнеры с помощью стеганографических методов [6]. Одним из самых распространенных и самых простых стеганографических методов является так называемый LSB-метод (Least Significant Bit-метод). Его принцип состоит в следующем. Берется некоторый цифровой мультимедиа-контейнер, представляющий собой файл с цифровым изображением, аудио или видео, и наименее значимые биты отсчетов данного контейнера заменяются на биты некоторого сообщения. Так как современные цифровые мультимедиа-форматы достаточно избыточны по своей структуре, при изменении определенного количества таких бит (то есть по сути внесения шума) качество мультимедиа-данных при восприятии их человеком практически не меняется.

Одним из самых известных легальных способов применения стеганографических методов являются цифровые водяные знаки (digital watermarks) [7], которые позволяют встраивать в мультимедиа-данные различную дополнительную информацию, например, об авторских правах на аудио-композицию или о пациенте, чей цифровой ренттеновский снимок хранится в базе данных больницы. Другая сторона использования стеганографии - скрытая передача информации, когда в глобальных сетях пересылаются внешне безобидные данные, например, картинка с изображением Эйфелевой башни, в которую 
встроено сообщение, например, о нелегальной продаже оружия. В современном мире проблема международного терроризма стоит очень остро, при этом уже ни для кого не секрет, что современные глобальные сети представляют собой очень удобный механизм для связи террористических организаций по всему миру. Стеганографические методы позволяют осуществлять такую связь скрыто от любых контролирующих органов. Поэтому задача выявления случаев скрытой передачи информации в настоящее время стоит весьма остро.

Раздел 2 данной работы посвящен описанию модели дискретного марковского поля, а также упрощенной модели, используемой в дальнейшем. Раздел 3 содержит описание модели скрытого марковского поля в случае LSB-модификации исходного марковского поля, описывающего немодифицированное изображение. Далее рассматривается подход, который упрощает вычисление апостериорных вероятностей СММ, основанный на условной теореме Байеса. В конще второй части данный подход применяется совместно с известным ЕМ-алгоритмом для оценки параметров скрытого марковского поля.

\section{2. Дискретные марковские поля}

Рассмотрим модель дискретного марковского поля, которая традиционно используется для описания изображения. Пусть $S=\{s\}$ представляет собой конечный набор индексов, соответствующий пикселям изображения. Множество

$$
\mathbf{X}=\prod_{s \in S} \mathbf{X}_{s}
$$

представляет собой конечный набор возможных значений цветов пикселей изображения, где $\mathbf{X}_{s}=\left\{x_{s}\right\}-$ конечное множество значений цветов. Будем называть $x=\left(x_{s}\right)_{s \in S}$ конфигурацией из множества $\mathbf{X}$. Обычно $S=\{s\}$ представляет собой прямоугольную решетку, то есть $s=(i, j)$, где $1 \leqslant i \leqslant W, 1 \leqslant j \leqslant H$, при этом $x$ описывает значения цветов пикселей некоторого прямоугольного изображения.

Определение 1. Пусть $(\Omega, \mathscr{F}, \mathbf{P})$ - вероятностное пространство, $(\mathbf{X}, \sigma(\mathbf{X}))$ - измеримое пространство, где $\sigma(\mathbf{X})$ - сигма-алгебра всех подмножеств $\mathbf{X}$. Семейство случайных величин

$$
X(\omega)=\left(X_{s}(\omega)\right)_{s \in S}, \quad X_{s}: \Omega \rightarrow \mathbf{X}_{s}
$$

такое, что

$$
\mathbf{P}(\omega: X(\omega)=x)=\mathbf{P}(X=x)=\Pi(x)>0,
$$

представляет собой случайное поле.

Распределение П случайного поля $X$ можно представить в гиббсовской форме [1]:

$$
\Pi(x)=\frac{\exp (-H(x))}{\sum_{z \in \mathrm{X}} \exp (-H(z))},
$$

где $H(x)$ - энергетическая функия. Для некоторого подмножества индексов (группы пикселей) $A \subset S$ можно определить

$$
\mathbf{X}_{A}=\prod_{s \in A} \mathbf{X}_{s}, \quad x_{A}=\left(x_{s}\right)_{s \in A}
$$


Тогда

$$
\begin{aligned}
\mathbf{P}\left(X_{A}=x_{A} \mid X_{S \backslash A}=x_{S \backslash A}\right) & =\mathbf{P}(X=x) / \mathbf{P}\left(X_{S \backslash A}=x_{S \backslash A}\right) \\
& =\mathbf{P}(X=x) / \sum_{z_{A} \in \mathbf{X}_{\mathbf{A}}} \mathbf{P}\left(X=z_{A} x_{S \backslash A}\right) \\
& =\Pi(x) / \sum_{z_{A}} \Pi\left(z_{A} x_{S \backslash A}\right) .
\end{aligned}
$$

Определение 2. Система подмножеств $\delta=\{\delta(s) \subset S, s \in S\}$ называется системой соседей, если $s \notin \delta(s)$ и $s \in \delta(t) \Longleftrightarrow t \in \delta(s)$. Множество $C \subset S$ называется кликой, если для любых $s$ и $t$ из $C$ справедливо включение $s \in \delta(t)$ (или $t \in \delta(s)$ ), то есть $s$ и $t$ являются соседними относительно $\delta$.

Определение 3. Случайное поле $X$ называется марковским с системой соседей $\delta$, если

$$
\mathbf{P}\left(X_{t}=x_{t} \mid X_{S \backslash\{t\}}=x_{S \backslash\{t\}}\right)=\mathbf{P}\left(X_{t}=x_{t} \mid X_{\delta(t)}=x_{\delta(t)}\right) .
$$

Справедлива следующая теорема Хаммерсли-Клиффорда.

Теорема 1 ([2]). Для марковского случайного поля с системой окрестностей $\delta$

$$
H(x)=\sum_{C \subset S} U_{C}\left(x_{C}\right)
$$

где $U_{C}: \mathbf{X}_{C} \rightarrow \mathbf{R}-$ энергетический потенчиал клики $C$,

$$
\mathbf{P}\left(X_{t}=x_{t} \mid X_{\delta(t)}=x_{\delta(t)}\right)=\frac{\exp \left(-\sum_{C \ni t} U_{C}\left(x_{C}\right)\right)}{\sum_{z_{t}} \exp \left(-\sum_{C \ni t} U_{C}\left(x_{C \backslash\{t\}} z_{t}\right)\right)} .
$$

Такая математическая модель описывает зависимости между соседними пикселями и упрощает анализ изображений.

Далее будет рассматриваться система соседей, в которой соседними считаются пиксели, ближайшие по вертикали и по горизонтали (в форме креста), в случае, когда $S$ представляет собой прямоугольную решетку

$$
\delta(s)=\delta(i, j)=\left\{\left(i_{1}, j_{1}\right) \in S:\left|i-i_{1}\right|+\left|j-j_{1}\right|=1\right\} .
$$

Все клики, образованные такой системой соседей, состоят из пар соседних пикселей.

Если рассматриваемое марковское поле однородное, то в этом случае

$$
U_{C}(\cdot)=U_{\{s, t\}}(\cdot)=U(\cdot)
$$

не зависит от $s$ и $t$, и тогда

$$
\begin{aligned}
H(x) & =\sum_{\{s, t\} \subset S} U\left(x_{s}, x_{t}\right), \\
\Pi(x) & =\frac{1}{Z} \prod_{t \in S} b\left(x_{t}, x_{\delta(t)}\right) \\
h\left(x_{t}, x_{\delta(t)}\right) & =\prod_{s \in \delta(t)} \exp \left(-(1 / 2) U\left(x_{t}, x_{s}\right)\right)
\end{aligned}
$$


где $Z$ - нормирующая константа.

Будем считать, без потери общности, что $\mathbf{X}_{s}=\left\{e_{1}, \ldots, e_{N}\right\}$ для любого $s \in S$, где $e_{i}$ - единичный вектор из пространства $\mathbf{R}^{N}$, у которого все компоненты, кроме $i$-й, равны 0 , а $i$-я компонента равна 1 .

Используя теорему Хаммерсли-Клиффорда и (1), можно показать, что

$$
\begin{aligned}
\mathbf{P}\left(X_{t}=x_{t} \mid X_{\delta(t)}=x_{\delta(t)}\right) & =\frac{\mathbf{P}\left(X_{t \delta(t)}=x_{t \delta(t)}\right)}{\sum_{z_{t} \in \mathbf{X}_{t}} \mathbf{P}\left(X_{t \delta(t)}=z_{t} x_{\delta(t)}\right)}=\frac{\exp \left(-\sum_{\{t, s\} \ni t} U\left(x_{t}, x_{s}\right)\right)}{\sum_{z_{t}} \exp \left(-\sum_{\{t, s\} \ni t} U\left(z_{t}, x_{s}\right)\right)} \\
& =\frac{\prod_{s \in \delta(t)} \exp \left(-U\left(x_{t}, x_{s}\right)\right)}{\sum_{z_{t}} \prod_{s \in \delta(t)} \exp \left(-U\left(z_{t}, x_{s}\right)\right)}=\frac{b\left(x_{t}, x_{\delta(t)}\right)^{2}}{\sum_{z_{t}} b\left(z_{t}, x_{\delta(t)}\right)^{2}} \\
& =a^{x_{t}}\left(x_{\delta(t)}\right) .
\end{aligned}
$$

Тогда для неграничных пикселей изображения, то есть для тех $t \in S$, для которых $|\delta(t)|=4$, вероятности $\mathbf{P}\left(X_{t}=x_{t} \mid X_{\delta(t)}=x_{\delta(t)}\right)$ могут быть заданы с помощью пятимерной матрицы

$$
A=\left\{a^{e_{n}}\left(e_{k_{1}}, e_{k_{2}}, e_{k_{3}}, e_{k_{4}}\right), 1 \leqslant n, k_{i} \leqslant N\right\}
$$

(аналога матрицы переходных вероятностей для марковской цепи). Для граничных пикселей данные условные вероятности будут задаваться матрицами меньших порядков (3-х и 2-хмерными).

Лемма 1. Для неграничных пикселей изображения

$$
X_{t}=A\left(X_{\delta(t)}\right)+V_{t}, \quad \mathbf{E}\left[V_{t} \mid \mathscr{F}_{S \backslash\{t\}}\right]=0,
$$

где операчия $A\left(z_{\delta(t)}\right), z_{\delta(t)} \in \mathbf{X}_{\delta(t)}$, определяет вектор из $\mathbf{R}^{N}$, состоящий из элементов матрицы $A:\left(a^{e_{1}}\left(z_{\delta(t)}\right), \ldots, a^{e_{N}}\left(z_{\delta(t)}\right)\right)^{T} ; \mathscr{F}_{S \backslash\{t\}}-$ сигма-алгебра, порожденная случайными величинами $X_{S \backslash\{t\}}$.

Доказательство. Нетрудно видеть, что

$$
\begin{aligned}
\mathbf{E}\left[V_{t} \mid \mathscr{F}_{S \backslash\{t\}}\right] & =\mathbf{E}\left[X_{t}-A\left(X_{\delta(t)}\right) \mid \mathscr{F}_{S \backslash\{t\}}\right]=\mathbf{E}\left[X_{t} \mid \mathscr{F}_{S \backslash\{t\}}\right]-A\left(X_{\delta(t)}\right) \\
& =A\left(X_{\delta(t)}\right)-A\left(X_{\delta(t)}\right)=0 .
\end{aligned}
$$

Лемма доказана.

Аналогичные леммы могут быть доказаны и для граничных пикселей изображения.

\section{3. Скрытое марковское поле}

Скрытые марковские модели используются в случаях, когда исходное марковское поле $X$ недоступно для наблюдателя, а доступно его некоторая случайная модификация $Y$ (см. $[3,2])$, причем случайное поле $Y$ определено на соответствующем конечном множестве

$$
\mathbf{Y}=\prod_{s \in S} \mathbf{Y}_{\boldsymbol{s}}
$$


И

$$
\mathrm{E}\left[Y_{t} \mid \mathscr{F}_{S}, \mathscr{Y}_{S \backslash\{t\}}\right]=\mathrm{E}\left[Y_{t} \mid X_{t}\right]
$$

где

$$
\mathscr{F}_{S}=\sigma\left\{X_{s}, s \in S\right\}, \quad \mathscr{Y}_{S \backslash\{t\}}=\sigma\left\{Y_{s}, s \in S, s \neq t\right\} .
$$

Таким образом, вся априорная информация о значении $Y_{t}$ случайного поля $Y$ в узле решетки $t$ содержится в значении исходного марковского случайного поля $X$ в данном узле решетки.

В случаe LSB-модификации исходного марковского поля (изображения)

$$
\mathbf{Y}=\mathbf{X}=\prod_{s \in S}\left\{e_{1}, \ldots, e_{N}\right\}
$$

и

$$
\mathbf{P}\left(Y_{t}=e_{k} \mid X_{t}=e_{m}\right)= \begin{cases}1-\theta, & \text { если } \bar{e}_{k}=e_{m}, \\ \theta, & \text { если } e_{k}=e_{m}, \\ 0 & \text { при остальных } e_{k}, e_{m},\end{cases}
$$

где $0<\theta \leqslant 1$ и $\bar{e}_{k}-$ операция изменения последнего бита, $\bar{e}_{k}=e_{k+1}$, если $k$ четно и $\bar{e}_{k}=e_{k-1}$, если $k$ нечетно. Параметр $\theta$ - это вероятность того, что при LSB-модификации последний бит значения цвета некоторого пикселя изображения не изменится. Такая модель традиционно рассматривается в задачах стегоанализа, при этом, чем меньше $\theta$, тем больше количество встраиваемой информации [8]. Операция изменения последнего бита определяется вышеописанным образом, исходя из того, что при двоичном представлении четных чисел их последний бит равен 0 , а при двоичном представлении нечетных - 1 . По сути, основная задача стегоанализа состоит в оценке параметра $\theta$ по информации из $\mathscr{Y}_{S}=\sigma\left\{Y_{s}, s \in S\right\}$.

Лемма 2. Для любого $t \in S$

$$
Y_{t}=\theta X_{t}+(1-\theta) \bar{X}_{t}+W_{t}, \quad \mathrm{E}\left[W_{t} \mid \mathscr{Y}_{S \backslash\{t\}}\right]=0 .
$$

Доказательство. Нетрудно видеть, что

$$
\begin{aligned}
\mathrm{E}\left[W_{t} \mid \mathscr{Y}_{S \backslash\{t\}}\right] & =\mathrm{E}\left[Y_{t}-\theta X_{t}-(1-\theta) \bar{X}_{t} \mid \mathscr{Y}_{S \backslash\{t\}}\right] \\
& =\mathrm{E}\left[\mathrm{E}\left[Y_{t}-\theta X_{t}-(1-\theta) \bar{X}_{t} \mid \mathscr{F}_{S}, \mathscr{Y}_{S \backslash\{t\}}\right] \mid \mathscr{Y}_{S \backslash\{t\}}\right] \\
& =\mathrm{E}\left[\mathrm{E}\left[Y_{t} \mid X_{t}\right]-\theta X_{t}-(1-\theta) \bar{X}_{t} \mid \mathscr{Y}_{S \backslash\{t\}}\right] \\
& =\mathrm{E}\left[\theta X_{t}+(1-\theta) \bar{X}_{t}-\theta X_{t}-(1-\theta) \bar{X}_{t} \mid \mathscr{Y}_{S \backslash\{t\}}\right]=0 .
\end{aligned}
$$

Лемма доказана.

Итак, скрытая марковская модель в случае LSB-модификации дискретного марковского поля описывается набором уравнений

$$
\begin{gathered}
X_{t}=A\left(X_{\delta(t)}\right)+V_{t}, \quad Y_{t}=\theta X_{t}+(1-\theta) \bar{X}_{t}+W_{t}, \\
\mathbf{E}\left[V_{t} \mid \mathscr{F}_{S \backslash\{t\}}\right]=0, \quad \mathbf{E}\left[W_{t} \mid \mathscr{Y}_{S \backslash\{t\}}\right]=0 .
\end{gathered}
$$




\section{1. Изменение меры для поля $Y$}

Здесь и далее применяется методика изменения меры, которая была предложена в [2] и опирается на аналог теоремы Гирсанова [9] для дискретного времени. Суть данной методики заключается в следующем: вводится новая вероятностная мера, для которой наблюдаемые величины являются независимыми (и может быть даже одинаково распределенными), вычисляются необходимые апостериорные вероятности, а затем полученные результаты переносятся на исходное вероятностное пространство с помощью обратного изменения меры.

Заметим, что $\mathbf{E}\left[\left\langle X_{t}, Y_{t}\right\rangle \mid X_{t}\right]=\theta$ и $\mathbf{E}\left[\left\langle X_{t}, \bar{Y}_{t}\right\rangle \mid X_{t}\right]=1-\theta$, для всех $t \in S$, где $\langle\cdot, \cdot\rangle$ - скалярное произведение в $\mathbf{R}^{N}$, при этом скалярные произведения $\left\langle X_{t}, Y_{t}\right\rangle$ и $\left\langle X_{t}, \bar{Y}_{t}\right\rangle$ принимают только значения 0 или 1 , то есть по сути являются индикаторами событий $\left\{X_{t}=Y_{t}\right\}$ и $\left\{X_{t}=\bar{Y}_{t}\right\}$. Введем случайные величины

$$
\begin{aligned}
\lambda_{t} & =\frac{1}{2}\left(\frac{1}{\theta}\right)^{\left\langle X_{t}, Y_{t}\right\rangle}\left(\frac{1}{1-\theta}\right)^{\left\langle X_{t}, \bar{Y}_{t}\right\rangle}=\frac{1}{2}\left(\frac{\left\langle X_{t}, Y_{t}\right\rangle}{\theta}+\frac{\left\langle X_{t}, \bar{Y}_{t}\right\rangle}{1-\theta}\right), \quad t \in S, \\
\Lambda_{S} & =\prod_{t \in S} \lambda_{t} .
\end{aligned}
$$

Лемма 3. Справедливо равенство

$$
\mathrm{E}\left[\lambda_{t} \mid \mathscr{F}_{S}, \mathscr{Y}_{S \backslash\{t\}}\right]=1 .
$$

Доказательство. Нетрудно видеть, что

$$
\begin{aligned}
\mathbf{E}\left[\lambda_{t} \mid \mathscr{F}_{S}, \mathscr{Y}_{S \backslash\{t\}}\right] & =\frac{1}{2} \mathbf{E}\left[\left(\frac{\left\langle X_{t}, Y_{t}\right\rangle}{\theta}+\frac{\left\langle X_{t}, \bar{Y}_{t}\right\rangle}{1-\theta}\right) \mid \mathscr{F}_{S}, \mathscr{Y}_{S \backslash\{t\}}\right] \\
& =\frac{1}{2}\left(\frac{\mathbf{E}\left[\left\langle X_{t}, Y_{t}\right\rangle \mid X_{t}\right]}{\theta}+\frac{\mathbf{E}\left[\left\langle X_{t}, \bar{Y}_{t}\right\rangle \mid X_{t}\right]}{1-\theta}\right)=\frac{1}{2}(1+1)=1 .
\end{aligned}
$$

Лемма доказана.

Используя данную лемму, нетрудно показать, что $\mathbf{E}\left[\Lambda_{S}\right]=1$.

На измеримом пространстве $\left(\mathbf{X}, \mathscr{F}_{S} \vee \mathscr{Y}_{S}\right)$ определим новую вероятностную меру

$$
\overline{\mathbf{P}}(B)=\int_{B} \Lambda_{S} d \mathbf{P}, \quad B \in \mathscr{F}_{S} \vee \mathscr{Y}_{S},
$$

в этом случае $d \overline{\mathbf{P}} / d \mathbf{P}=d \overline{\mathbf{P}} /\left.d \mathbf{P}\right|_{\mathscr{F}_{S} \vee_{y_{S}}}=\Lambda_{S}$ (производная Радона-Никодима).

Справедлива следующая условная Байесовская теорема.

Теорема 2 ([2]). Пусть $(\Omega, \mathscr{F}, \mathbf{P})$ - вероятностное пространство, $\mathscr{G} \subset \mathscr{F}$ - сигмаалгебра, $\overline{\mathbf{P}}$ - вероятностная мера на $(\Omega, \mathscr{F})$, абсолютно непрерывная относительно $\mathbf{P}$, с производной Радона-Никодима $d \overline{\mathbf{P}} / d \mathbf{P}=\Lambda$.

Тогда для любой F्F-измеримой случайной величины $\varphi$ такой, что $\mathrm{E}[\varphi]<\infty$, справедливо равенство

$$
\overline{\mathbf{E}}[\varphi \mid \mathscr{G}]=\frac{\mathbf{E}[\varphi \Lambda \mid \mathscr{G}]}{\mathbf{E}[\varphi \mid \mathscr{G}]}
$$

где $\overline{\mathbf{E}}$ - математическое ожидание по мере $\overline{\mathbf{P}}$. 
Данная теорема будет использована при доказательстве следующей теоремы.

Теорема 3. Для всех $t \in S$

$$
\overline{\mathbf{P}}\left[X_{t}=Y_{t} \mid \mathscr{F}_{S}, \mathscr{Y}_{S \backslash\{t\}}\right]=\overline{\mathbf{P}}\left[X_{t}=\bar{Y}_{t} \mid \mathscr{F}_{S}, \mathscr{Y}_{S \backslash\{t\}}\right]=1 / 2,
$$

то есть по мере $\overline{\mathbf{P}}$ апостериорная вероятность изменения последнего бита равна $1 / 2$. Доказательство. Нетрудно видеть, что

$$
\begin{aligned}
\overline{\mathbf{E}}\left[\left\langle X_{t}, Y_{t}\right\rangle \mid \mathscr{F}_{S}, \mathscr{Y}_{S \backslash\{t\}}\right] & =\frac{\mathbf{E}\left[\left\langle X_{t}, Y_{t}\right\rangle \Lambda_{S} \mid \mathscr{F}_{S}, \mathscr{Y}_{S \backslash\{t\}}\right]}{\mathbf{E}\left[\Lambda_{S} \mid \mathscr{F}_{S}, \mathscr{Y}_{S \backslash\{t\}}\right]}=\frac{\Lambda_{S \backslash\{t\}} \mathbf{E}\left[\left\langle X_{t}, Y_{t}\right\rangle \lambda_{t} \mid \mathscr{F}_{S}, \mathscr{Y}_{S \backslash\{t\}}\right]}{\Lambda_{S \backslash\{t\}} \mathbf{E}\left[\lambda_{t} \mid \mathscr{F}_{S}, \mathscr{Y}_{S \backslash\{t\}}\right]} \\
& =\mathbf{E}\left[\frac{\left\langle X_{t}, Y_{t}\right\rangle}{2}\left(\frac{\left\langle X_{t}, Y_{t}\right\rangle}{\theta}+\frac{\left\langle X_{t}, \bar{Y}_{t}\right\rangle}{1-\theta}\right) \mid \mathscr{F}_{S}, \mathscr{Y}_{S \backslash\{t\}}\right] \\
& =\mathbf{E}\left[\frac{\left\langle X_{t}, Y_{t}\right\rangle^{2}}{2 \theta}+\frac{\left\langle X_{t}, Y_{t}\right\rangle\left\langle X_{t}, \bar{Y}_{t}\right\rangle}{2(1-\theta)} \mid \mathscr{F}_{S}, \mathscr{Y}_{S \backslash\{t\}}\right] \\
& =\frac{\mathbf{E}\left[\left\langle X_{t}, Y_{t}\right\rangle^{2} \mid \mathscr{F}_{S}, \mathscr{Y}_{S \backslash\{t\}}\right]}{2 \theta}+0=\frac{\theta}{2 \theta}=\frac{1}{2},
\end{aligned}
$$

так как $\left\langle X_{t}, Y_{t}\right\rangle\left\langle X_{t}, \bar{Y}_{t}\right\rangle$ всегда равно нулю, как произведение индикаторов несовместных событий, и $\left\langle X_{t}, Y_{t}\right\rangle^{2}=\left\langle X_{t}, Y_{t}\right\rangle$, потому что $\left\langle X_{t}, Y_{t}\right\rangle$ принимает только значения 0 и 1. Аналогично доказывается, что

$$
\overline{\mathbf{E}}\left[\left\langle X_{t}, \bar{Y}_{t}\right\rangle \mid \mathscr{F}_{S}, \mathscr{Y}_{S \backslash\{t\}}\right]=1 / 2 .
$$

Теорема доказана.

\section{2. Изменение меры для поля $X$}

Обозначим $\hat{\varphi}(\cdot)$ равномерное распределение на $\mathbf{X}_{s}, s \in S$, такое, что $\hat{\varphi}\left(e_{i}\right)=1 / N$, $1 \leqslant i \leqslant N$. Положим

$$
\widehat{\Phi}(x)=\prod_{t \in S} \hat{\varphi}\left(x_{t}\right)=\left(\frac{1}{N}\right)^{|S|} .
$$

Возьмем $\Gamma_{S}(x)=\widehat{\Phi}(x) / \mathbf{P}(x)\left(\mathbf{E}\left[\Gamma_{S}(X)\right]=1\right)$ и определим меру $\hat{\mathbf{P}}$ на измеримом пространстве $\left(\mathbf{X}, \mathscr{F}_{S} \vee \mathscr{Y}_{S}\right)$ такую, что

$$
\frac{d \hat{\mathbf{P}}}{d \overline{\mathbf{P}}}=\left.\frac{d \hat{\mathbf{P}}}{d \overline{\mathbf{P}}}\right|_{\mathscr{F}_{S} \vee \mathscr{Q}_{S}}=\Gamma_{S}(X)=\Gamma_{S} .
$$

Лемма 4. Случайные величины $X_{t}, t \in S$, независимы и одинаково равномерно на $\mathbf{X}_{t}$ распределены относительно меры $\hat{\mathbf{P}}$.

Доказательство. Нетрудно видеть, что

$$
\begin{aligned}
E_{t, m} & =\hat{\mathbf{E}}\left[\left\langle X_{t}, e_{m}\right\rangle \mid \mathscr{F}_{S \backslash\{t\}}\right]=\overline{\mathbf{E}}\left[\left\langle X_{t}, e_{m}\right\rangle \Gamma_{S} \mid \mathscr{F}_{S \backslash\{t\}}\right] / \overline{\mathbf{E}}\left[\Gamma_{S} \mid \mathscr{F}_{S \backslash\{t\}}\right] \\
& =\overline{\mathbf{E}}\left[\left\langle X_{t}, e_{m}\right\rangle \widehat{\Phi}(X) / \mathbf{P}(X) \mid \mathscr{F}_{S \backslash\{t\}}\right] / \overline{\mathbf{E}}\left[\widehat{\Phi}(X) / \mathbf{P}(X) \mid \mathscr{F}_{S \backslash\{t\}] .}\right.
\end{aligned}
$$


Сокращая все множители, которые не зависят от $X_{t}$, и продолжая преобразования, получаем, что

$$
\begin{aligned}
E_{t, m}= & \frac{\overline{\mathbf{E}}\left[\frac{\left\langle X_{t}, e_{m}\right) \hat{\varphi}\left(X_{t}\right)}{\prod_{s \in \overline{\delta(t)}} b\left(X_{s}, X_{\delta(s)}\right)} \mid \mathscr{F}_{S \backslash\{t\}}\right]}{\overline{\mathbf{E}}\left[\frac{\hat{\varphi}\left(X_{t}\right)}{\prod_{s \in \overline{\delta(t)}} b\left(X_{s}, X_{\delta(s)}\right)} \mid \mathscr{F}_{S \backslash\{t\}}\right]}=\frac{\overline{\mathbf{E}}\left[\frac{\left\langle X_{t}, e_{m}\right\rangle}{\prod_{s \in \delta(t)} \exp \left(-U\left(X_{s}, X_{t}\right)\right.} \mid \mathscr{F}_{S \backslash\{t\}}\right]}{\overline{\mathbf{E}}\left[\frac{1}{\prod_{s \in \delta(t)} \exp \left(-U\left(X_{s}, X_{t}\right)\right.} \mid \mathscr{F}_{S \backslash\{t\}}\right]} \\
= & \frac{\left(\overline{\mathrm{E}}\left[\left\langle X_{t}, e_{m}\right\rangle \mid \mathscr{F}_{S \backslash\{t\}}\right] / \Pi_{m}\right)}{\sum_{p=1}^{N}\left(\overline{\mathbf{E}}\left[\left\langle X_{t}, e_{p}\right\rangle \mid \mathscr{F}_{S \backslash\{t\}}\right] / \Pi_{p}\right)}=\frac{A_{m}}{\sum_{p=1}^{N} A_{p}},
\end{aligned}
$$

где

$$
\Pi_{p}=\prod_{s \in \delta(t)} \exp \left(-U\left(X_{s}, e_{k}\right)\right), \quad A_{p}=\frac{1}{\Pi_{p}} \frac{\Pi_{p}}{\sum_{k=1}^{N} \Pi_{k}}, \quad p=1, \ldots, N
$$

Нетрудно видеть, что правая часть этого равенства есть $1 / N$, так как

$$
\begin{aligned}
\prod_{s \in \hat{\delta(t)}} b\left(X_{s}, X_{\delta(s)}\right) & =\prod_{s \in \overline{\delta(t)}} \prod_{l \in \delta(s)} \exp \left(-(1 / 2) U\left(X_{l}, X_{s}\right)\right) \\
& =\prod_{s \in \delta(t)} \exp \left(-U\left(X_{s}, X_{t}\right)\right) \prod_{s, l \neq t} \exp \left(-(1 / 2) U\left(X_{l}, X_{s}\right)\right)
\end{aligned}
$$

где, как и выше, $\overline{\delta(t)}=\delta(t) \cup t$.

Лемма доказана.

Таким образом, можно изначально рассматривать вероятностную меру $\hat{\mathbf{P}}$ на измеримом пространстве $\left(\mathbf{X}, \mathscr{F}_{S} \vee \mathscr{Y}_{S}\right)$ такую, что все $X_{t}$ будут независимы и одинаково равномерно распределены на $\mathbf{X}_{t}$. Тогда по заданным функциям $U\left(x_{t}, x_{s}\right)$ (многомерной матрице $A$ ) можно построить вероятностную меру $\overline{\mathbf{P}}$ такую, что

$$
\begin{aligned}
\overline{\mathbf{P}}\left[X_{t}=x_{t} \mid X_{S \backslash\{t\}}=x_{S \backslash\{t\}}\right] & =\overline{\mathbf{P}}\left[X_{t}=x_{t} \mid X_{\delta(t)}=x_{\delta(t)}\right]=\mathbf{P}\left[X_{t}=x_{t} \mid X_{\delta(t)}=x_{\delta(t)}\right] \\
& =\frac{\prod_{s \in \delta(t)} \exp \left(-U\left(x_{s}, x_{t}\right)\right)}{\sum_{z_{t}} \prod_{s \in \delta(t)} \exp \left(-U\left(x_{s}, z_{t}\right)\right)}=a^{x_{t}}\left(x_{\delta(t)}\right) .
\end{aligned}
$$

Меру $\overline{\mathbf{P}}$ можно определить так, что $d \overline{\mathbf{P}} / d \widehat{\mathbf{P}}=\bar{\Gamma}_{S}$, где

$$
\bar{\Gamma}_{S}=\Gamma_{S}^{-1}=\frac{\mathbf{P}(x)}{\widehat{\Phi}(x)},
$$

так как для двух эквивалентных $\sigma$-конечных мер $\mu$ и $\nu$ справедливо равенство $d \mu / d \nu=(d \nu / d \mu)^{-1}[10]$, а эквивалентность $\overline{\mathbf{P}}_{\text {и }} \hat{\mathbf{P}}$ следует из того, что $\Gamma_{S}>0$.

Аналогичным образом можно изначально рассматривать вероятностную меру $\hat{\mathbf{P}}$ на $\left(\mathbf{X}, \mathscr{F}_{S} \vee \mathscr{Y}_{S}\right)$ такую, что оба случайных поля $X_{t}, t \in S$, и $Y_{t}, t \in S$, независимы и одинаково равномерно распределены на $\mathbf{X}_{t}, t \in S$. Получить требуемую меру $\mathbf{P}$ можно, положив

$$
\frac{d \mathbf{P}}{d \hat{\mathbf{P}}}=\frac{d \mathbf{P}}{d \overline{\mathbf{P}}} \frac{d \overline{\mathbf{P}}}{d \hat{\mathbf{P}}}=\bar{\Lambda}_{S} \bar{\Gamma}_{S}
$$




\section{3. Рекурсия}

Будем рассматривать измеримое пространство $\left(\mathbf{X}, \mathscr{F}_{S} \vee \mathscr{Y}_{S}\right)$ с вероятностной мерой $\hat{\mathbf{P}}$, учитывая что согласно условной теореме Байеса

$$
\mathbf{E}\left[\left\langle X_{t}, Y_{t}\right\rangle \mid \mathscr{Y}_{S}\right]=\frac{\hat{\mathbf{E}}\left[\left\langle X_{t}, Y_{t}\right\rangle \bar{\Lambda}_{S} \bar{\Gamma}_{S} \mid \mathscr{Y}_{S}\right]}{\hat{\mathbf{E}}\left[\bar{\Lambda}_{S} \bar{\Gamma}_{S} \mid \mathscr{Y}_{S}\right]}
$$

Определение 4. Обозначим

$$
q_{t}=\hat{\mathrm{E}}\left[\left\langle X_{t}, Y_{t}\right\rangle \bar{\Lambda}_{S} \bar{\Gamma}_{S} \mid \mathscr{Y}_{S}\right], \quad t \in S,
$$

ненормализованную апостериорную вероятность того, что последний бит $X_{t}$ не был изменен. Аналогично обозначим

$$
q_{\bar{t}}=\hat{\mathbf{E}}\left[\left\langle X_{t}, \bar{Y}_{t}\right\rangle \bar{\Lambda}_{S} \bar{\Gamma}_{S} \mid \mathscr{Y}_{S}\right], \quad t \in S .
$$

Теорема 4. Для всех $t \in S$ ненормализованные вероятности $q_{t} u q_{\bar{t}}$ определяются в соответствии со следующими псевдорекурсивными уравнениями:

$$
q_{t}=\frac{2}{N} \theta \beta_{S \backslash\{t\}}, \quad q_{\bar{t}}=\frac{2}{N}(1-\theta) \beta_{S \backslash\{\bar{t}\}},
$$

2de

$$
\begin{aligned}
& \beta_{S \backslash\{t\}}=\hat{\mathbf{E}}\left[\bar{\Lambda}_{S \backslash\{t\}} \bar{\Gamma}_{S} \mid X_{t}=Y_{t}, \mathscr{Y}_{S}\right] \\
& \beta_{S \backslash\{\bar{t}\}}=\hat{\mathbf{E}}\left[\bar{\Lambda}_{S \backslash\{t\}} \bar{\Gamma}_{S} \mid X_{t}=\bar{Y}_{t}, \mathscr{Y}_{S}\right]
\end{aligned}
$$

Доказательство. Нетрудно видеть, что

$$
\begin{aligned}
q_{t} & =\hat{\mathbf{E}}\left[\left\langle X_{t}, Y_{t}\right\rangle \bar{\Lambda}_{S} \bar{\Gamma}_{S} \mid \mathscr{Y}_{S}\right]=\hat{\mathbf{E}}\left[\left\langle X_{t}, Y_{t}\right\rangle 2 \theta \bar{\Lambda}_{S \backslash\{t\}} \bar{\Gamma}_{S} \mid \mathscr{Y}_{S}\right] \\
& =2 \theta \hat{\mathbf{E}}\left[\left\langle X_{t}, Y_{t}\right\rangle \hat{\mathbf{E}}\left[\bar{\Lambda}_{S \backslash\{t\}} \bar{\Gamma}_{S} \mid X_{t}=Y_{t}, \mathscr{Y}_{S}\right] \mid \mathscr{Y}_{S}\right] \\
& =2 \theta \hat{\mathbf{E}}\left[\bar{\Lambda}_{S \backslash\{t\}} \bar{\Gamma}_{S} \mid X_{t}=Y_{t}, \mathscr{Y}_{S}\right] \hat{\mathbf{E}}\left[\left\langle X_{t}, Y_{t}\right\rangle \mid \mathscr{Y}_{S}\right]=\frac{2 \theta}{N} \beta_{S \backslash\{t\} .}
\end{aligned}
$$

Аналогичным образом получается выражение для $q_{\bar{t}}$.

Теорема доказана.

Теорема 5. Пусть

$$
\beta_{S \backslash\left\{t_{1}, \bar{t}_{2}, \ldots, t_{p}\right\}}=\hat{\mathbf{E}}\left[\bar{\Lambda}_{S \backslash\left\{t_{1}, t_{2}, \ldots, t_{p}\right\}} \bar{\Gamma}_{S} \mid X_{t_{1}}=Y_{t_{1}}, X_{t_{2}}=\bar{Y}_{t_{2}}, \ldots, X_{t_{p}}=Y_{t_{p}}, \mathscr{Y}_{S}\right]
$$

Тогда значение $\beta_{S \backslash\left\{t_{1}, \overline{t_{2}}, \ldots, t_{p}\right\}}$ может быть получено с помощью рекурсивного выражения

$$
\begin{aligned}
\beta_{S \backslash\left\{t_{1}, \overline{t_{2}}, \ldots, t_{p}\right\}} & =\frac{2}{N}\left(\theta \beta_{S \backslash\left\{t_{1}, \overline{t_{2}}, \ldots, t_{p}, s\right\}}+(1-\theta) \beta_{S \backslash\left\{t_{1}, \overline{t_{2}}, \ldots, t_{p}, \bar{s}\right\}}\right), s \in S \backslash\left\{t_{1}, \overline{t_{2}}, \ldots, t_{p}\right\}, \\
\beta_{S \backslash\left\{t_{1}, \overline{t_{2}}, \ldots, t_{|S|}\right\}} & =\Gamma_{S}\left(Y_{t_{1}}, \bar{Y}_{t_{2}}, \ldots, Y_{t_{|S|}}\right) .
\end{aligned}
$$

4 Дискретная математика, т.17 №3 
Доказательство. Проведем доказательство для первого и последнего шага рекурсии. Нетрудно видеть, что

$$
\begin{aligned}
& \beta_{S \backslash\{t\}}=\hat{\mathbf{E}}\left[\bar{\Lambda}_{S \backslash\{t\}} \bar{\Gamma}_{S} \mid X_{t}=Y_{t}, \mathscr{Y}_{S}\right] \\
& =\hat{\mathbf{E}}\left[2 \theta^{\left\langle X_{s}, Y_{s}\right\rangle}(1-\theta)^{\left\langle X_{s}, \bar{Y}_{S}\right\rangle} \hat{\mathbf{E}}\left[\bar{\Lambda}_{S \backslash\{t, s\}} \bar{\Gamma}_{S} \mid X_{t}=Y_{t}, X_{s}, \mathscr{Y}_{S}\right] \mid X_{t}=Y_{t}, \mathscr{Y}_{S}\right] \\
& =2 \theta \hat{\mathbf{E}}\left[\bar{\Lambda}_{S \backslash\{t, s\}} \bar{\Gamma}_{S} \mid X_{t}=Y_{t}, X_{s}=Y_{s}, \mathscr{Y}_{S}\right] \hat{\mathrm{E}}\left[\left\langle X_{s}, Y_{s}\right\rangle \mid X_{t}=Y_{t}, \mathscr{Y}_{S}\right] \\
& +2(1-\theta) \hat{\mathbf{E}}\left[\bar{\Lambda}_{S \backslash\{t, s\}} \bar{\Gamma}_{S} \mid X_{t}=Y_{t}, X_{s}=\bar{Y}_{s}, \mathscr{Y}_{S}\right] \\
& \times \hat{\mathrm{E}}\left[\left\langle X_{s}, \bar{Y}_{s}\right\rangle \mid X_{t}=Y_{t}, \mathscr{Y}_{S}\right] \\
& =\frac{2}{N}\left(\theta \beta_{S \backslash\{t, s\}}+(1-\theta) \beta_{S \backslash\{t, \bar{s}\}}\right), \\
& \beta_{S \backslash\left\{t_{1}, \bar{t}_{2}, \ldots, t_{|S|-1}\right\}}=\hat{\mathbf{E}}\left[\bar{\lambda}_{t_{|S|}} \bar{\Gamma}_{S} \mid X_{t_{1}}=Y_{t_{1}}, X_{t_{2}}=\bar{Y}_{t_{2}}, \ldots, X_{t_{|S|-1}}=Y_{t_{|S|-1}}, \mathscr{Y}_{S}\right] \\
& =\hat{\mathbf{E}}\left[2 \theta^{\left\langle X_{t_{|S|}}, Y_{t_{|S|}}\right\rangle}(1-\theta)^{\left\langle X_{t_{|S|}}, \bar{Y}_{t_{|S|}}\right\rangle}\right. \\
& \times \hat{\mathbf{E}}\left[\bar{\Gamma}_{S} \mid X_{t_{1}}=Y_{t_{1}}, \ldots, X_{t_{|S|-1}}=Y_{t_{|S|-1}}, X_{|S|}, \mathscr{Y}_{S}\right] \\
& \left.\mid X_{t_{1}}=Y_{t_{1}}, \ldots, X_{t_{|S|-1}}=Y_{t_{|S|-1}}, \mathscr{Y}_{S}\right] \\
& =\frac{2}{N}\left(\theta \hat{\mathbf{E}}\left[\bar{\Gamma}_{S} \mid X_{t_{1}}=Y_{t_{1}}, \ldots, X_{t_{|S|-1}}=Y_{t_{|S|-1}}, X_{|S|}=Y_{|S|}, \mathscr{Y}_{S}\right]\right. \\
& \left.+(1-\theta) \hat{\mathbf{E}}\left[\bar{\Gamma}_{S} \mid X_{t_{1}}=Y_{t_{1}}, \ldots, X_{t_{|S|-1}}=Y_{t_{|S|-1}}, X_{|S|}=\bar{Y}_{|S|}, \mathscr{Y}_{S}\right]\right) \\
& =\frac{2}{N}\left(\theta \beta_{S \backslash\left\{t_{1}, \bar{t}_{2}, \ldots, t_{|S|}\right\}}+(1-\theta) \beta_{S \backslash\left\{t_{1}, \bar{t}_{2}, \ldots, \bar{t}_{|S|}\right\}}\right) \\
& =\frac{2}{N}\left(\theta \Gamma_{S}\left(Y_{t_{1}}, \bar{Y}_{t_{2}}, \ldots, Y_{t_{|S|}}\right)+(1-\theta) \Gamma_{S}\left(Y_{t_{1}}, \bar{Y}_{t_{2}}, \ldots, \bar{Y}_{t_{|S|}}\right)\right) \text {. }
\end{aligned}
$$

Теорема доказана.

Теорема 6. Пусть $\mathbf{X} \mid$ у обозначает множество таких $x \in \mathbf{X}$, что для любого $t \in S$, либо $x_{t}=y_{t}$, либо $\bar{y}_{t}$, то есть множество $\mathbf{X} \mid y$ состоит из $x$, которые при замене некоторого числа последних бит могут преобразоваться в у. Тогда, для любого $x \in \mathbf{X} \mid Y$ справедливо равенство

$$
\mathbf{P}\left[X=x j \mathscr{y}_{S}\right]=\frac{\mathbf{P}(x) \theta^{\left|\left\{t: x_{t}=Y_{t}\right\}\right|}(1-\theta)^{\left|\left\{t: x_{t}=\bar{Y}_{t}\right\}\right|}}{\sum_{z \in \mathbf{X} \mid Y} \mathbf{P}(z) \theta^{\left|\left\{t: z_{t}=Y_{t}\right\}\right|}(1-\theta)^{\left|\left\{t: z_{t}=\bar{Y}_{t}\right\}\right|}} .
$$

Очевидно, что для любого $x \in \mathbf{X} \backslash \mathbf{X} \mid Y$ выполняется равенство $\mathbf{P}\left[X=x \mid \mathscr{y}_{S}\right]=0$. Доказательство. Нетрудно видеть, что

$$
\begin{aligned}
\mathbf{P}\left[X=x \mid \mathscr{Y}_{S}\right] & =\mathbf{E}\left[\prod_{t \in S}\left\langle X_{t}, x_{t}\right\rangle \mathscr{Y}_{S}\right]=\frac{\hat{\mathbf{E}}\left[\prod_{t \in S}\left\langle X_{t}, x_{t}\right\rangle \bar{\Lambda}_{S} \bar{\Gamma}_{S} \mid \mathscr{Y}_{S}\right]}{\hat{\mathbf{E}}\left[\bar{\Lambda}_{S} \bar{\Gamma}_{S} \mid \mathscr{Y}_{S}\right]} \\
& =\frac{\hat{\mathbf{E}}\left[\mathbf{P}(x) N^{|S|} 2^{|S|} \prod_{t \in S}\left\langle X_{t}, x_{t}\right\rangle \theta(1-\theta) \mid \mathscr{Y}_{S}\right]}{\hat{\mathbf{E}}\left[\mathbf{P}(X) N^{|S|} 2^{|S|} \prod_{t \in S} \theta(1-\theta) \mid \mathscr{Y}_{S}\right]} \\
& =\frac{\mathbf{P}(x) \theta^{\left|\left\{t: x_{t}=Y_{t}\right\}\right|}(1-\theta)^{\left|\left\{t: x_{t}=\bar{Y}_{t}\right\}\right|}}{\sum_{z \in \mathbf{X} \mid Y} \mathbf{P}(z) \theta^{\left|\left\{t: z_{t}=Y_{t}\right\}\right|}(1-\theta)^{\left|\left\{t: z_{t}=\bar{Y}_{t}\right\}\right|}}
\end{aligned}
$$

Теорема доказана. 
Для того, чтобы найти $x \in \mathbf{X} \mid Y$, максимизирующий апостериорную вероятность $\mathbf{P}\left[X=x \mid \mathscr{Y}_{S}\right]$, достаточно найти максимум функции

$$
\begin{aligned}
\mathscr{L}(x) & =\log \left[\mathbf{P}(x) \theta^{\left|\left\{t: x_{t}=Y_{t}\right\}\right|}(1-\theta)^{\left|\left\{t: x_{t}=\bar{Y}_{t}\right\}\right|}\right] \\
& =\log \mathbf{P}(x)+\left|\left\{t: x_{t}=Y_{t}\right\}\right| \log \theta+\left|\left\{t: x_{t}=\bar{Y}_{t}\right\}\right| \log (1-\theta) .
\end{aligned}
$$

\section{4. ЕМ-алгоритм}

ЕМ-алгоритм (Expectation-Maximization алгоритм) был предложен в [11]. Идея этого алгоритма состоит в следующем. Пусть $\left\{\mathbf{P}_{\theta}, \theta \in \Theta\right\}-$ семейство вероятностных мер на измеримом пространстве $(\Omega, \mathscr{F}), \mathbf{P}_{\theta} \ll \mathbf{P}_{0}$ для всех $\theta \in \Theta$. Для определения значения параметра $\theta$ по информации из сигма-алгебры У $\subset \mathscr{F}$ может быть использовано следующее условное отношение правдоподобия:

$$
L(\theta)=\mathbf{E}_{0}\left[\frac{d \mathbf{P}_{\theta}}{d \mathbf{P}_{0}} \mid \mathscr{y}\right],
$$

при этом оценка максимального правдоподобия определяется как

$$
\hat{\theta} \in \underset{\theta \in \Theta}{\operatorname{argmax}} L(\theta)
$$

В реальности прямое вычисление максимизирующего параметра редко представляется возможным, в [11] был предложен следующий итерационный метод аппроксимизации.

Шаг 1. Целочисленному параметру $p$ присваивается значение 0 и выбирается начальный параметр $\hat{\theta}_{0}$.

Шаг 2 (E-step). Задается значение $\theta^{*}=\hat{\theta}_{p}$ и вычисляется $Q\left(\cdot, \theta^{*}\right)$, где

$$
Q\left(\theta, \theta^{*}\right)=\mathbf{E}_{\theta^{*}}\left[\log \frac{d \mathbf{P}_{\theta}}{d \mathbf{P}_{\theta^{*}}} \mid \mathcal{Y}\right] .
$$

Шаг 3 (M-step). Определяется $\hat{\theta}_{p+1} \in \operatorname{argmax}_{\theta \in \Theta} Q\left(\theta, \theta^{*}\right)$.

Шаг 4. Полагается $p:=p+1$ и производится переход на Шаг 2 , если не выполнено условие остановки.

Последовательность параметров модели $\left\{\hat{\theta}_{p}, p \geqslant 0\right\}$ определяет неубывающий набор значений функции $L(\theta)$, что справедливо вследствие неравенства Иенсена, так как

$$
\log L\left(\hat{\theta}_{p+1}\right)-\log L\left(\hat{\theta}_{p}\right) \geqslant Q\left(\hat{\theta}_{p+1}, \hat{\theta}_{p}\right),
$$

при этом равенство выполнено тогда и только тогда, когда $\hat{\theta}_{p+1}=\hat{\theta}_{p}$. Функцию $Q\left(\theta, \theta^{*}\right)$ называют условной логарифмической функцией псевдоправдоподобия.

\section{5. Оценка параметров модели}

Параметрами скрытой марковской модели в описываемом случае являются $\{A, \theta\}$, где $A=\left\{a^{e_{n}}\left(e_{k_{1}}, e_{k_{2}}, e_{k_{3}}, e_{k_{4}}\right), 1 \leqslant n, k_{i} \leqslant N\right\}$. При этом

$$
\sum_{n=1}^{N} a^{e_{n}}\left(e_{k_{1}}, e_{k_{2}}, e_{k_{3}}, e_{k_{4}}\right)=1, \quad \theta>0
$$


Требуется найти набор параметров $\{\hat{A}, \hat{\theta}\}$, который бы максимизировал условную логарифмическую функцию псевдоправдоподобия при исходных значениях параметров $\{A, \theta\}$. Рассмотрим сначала вопрос оценки $\hat{\theta}$ параметра $\theta$. Определим

$$
\psi_{t}=\left(\frac{\hat{\theta}}{\theta}\right)^{\left\langle X_{t}, Y_{t}\right\rangle}\left(\frac{1-\hat{\theta}}{1-\theta}\right)^{\left\langle X_{t}, \bar{Y}_{t}\right\rangle}, \quad \Psi_{S}=\prod_{t \in S} \psi_{t} .
$$

Можно показать, что $\mathbf{E}\left[\Psi_{S}\right]=1$. Введем новую вероятностную меру $\hat{\mathbf{P}}$ такую, что $d \hat{\mathbf{P}} / d \mathbf{P}=\Psi_{S}$.

Лемма 5. Справедливы равенства

$$
\hat{\mathbf{P}}\left[X_{t}=Y_{t} \mid \mathscr{F}_{\mathscr{C}}, \mathscr{Y}_{S \backslash\{t\}}\right]=\hat{\theta}, \quad \hat{\mathbf{P}}\left[X_{t}=\bar{Y}_{t} \mid \mathscr{F}_{\mathscr{S}}, \mathscr{Y}_{S \backslash\{t\}}\right]=1-\hat{\theta} .
$$

Доказательство. Докажем первое равенство:

$$
\begin{aligned}
\hat{\mathbf{P}}\left[X_{t}\right. & \left.=Y_{t} \mid \mathscr{F}_{\mathscr{S}}, \mathscr{Y}_{S \backslash\{t\}}\right]=\hat{\mathbf{E}}\left[\left\langle X_{t}, Y_{t}\right\rangle \mid \mathscr{F}_{\mathscr{S}}, \mathscr{Y}_{S \backslash\{t\}}\right] \\
& =\frac{\mathbf{E}\left[\left\langle X_{t}, Y_{t}\right\rangle \Psi_{S} \mid \mathscr{F}_{\mathscr{S}}, \mathscr{Y}_{S \backslash\{t\}}\right]}{\mathbf{E}\left[\Psi_{S} \mid \mathscr{F}_{S}, \mathscr{Y}_{S \backslash\{t\}}\right]}=\frac{\mathbf{E}\left[\left\langle X_{t}, Y_{t}\right\rangle \psi_{t} \mid \mathscr{F}_{\mathscr{S}}, \mathscr{Y}_{S \backslash\{t\}}\right]}{\mathbf{E}\left[\psi_{t} \mid \mathscr{F}_{\mathscr{S}}, \mathscr{Y}_{S \backslash\{t\}}\right]} \\
& =\frac{(\hat{\theta} / \theta) \mathbf{E}\left[\left\langle X_{t}, Y_{t}\right\rangle^{2} \mid \mathscr{F}_{\mathscr{S}}, \mathscr{Y}_{S \backslash\{t\}}\right]}{(\hat{\theta} / \theta) \mathbf{E}\left[\left\langle X_{t}, Y_{t}\right\rangle \mid \mathscr{F}_{\mathscr{S}}, \mathscr{Y}_{S \backslash\{t\}}\right]+((1-\hat{\theta})(1-\theta)) \mathbf{E}\left[\left\langle X_{t}, \bar{Y}_{t}\right\rangle \mid \mathscr{F}_{\mathscr{S}}, \mathscr{Y}_{S \backslash\{t\}}\right]} \\
& =\frac{\hat{\theta}}{\hat{\theta}+(1-\hat{\theta})}=\hat{\theta} .
\end{aligned}
$$

Аналогично доказывается равенство для $\hat{\mathbf{P}}\left[X_{t}=\bar{Y}_{t} \mid \mathscr{F}_{\mathscr{S}}, \mathscr{Y}_{S \backslash\{t\}}\right]$.

Лемма доказана.

Теорема 7. Оченка условного максимального правдоподобия для параметра СММ $\theta$ при известных значениях $Y_{t}, t \in S$, на $M$-шаге определяется по формуле

$$
\hat{\theta}=\frac{\sum_{t \in S} q_{t}}{\sum_{t \in S}\left(q_{t}+q_{\bar{t}}\right)}
$$

где $q_{t}$ и $q_{\bar{t}}$ определены в (2).

Доказательство. Справедливы равенства

$$
\begin{aligned}
\mathbf{E}\left[\log \Psi_{S} \mid \mathscr{Y}_{S}\right] & =\mathbf{E}\left[\log \prod_{t \in S}\left(\frac{\hat{\theta}}{\theta}\right)^{\left\langle X_{t}, Y_{t}\right\rangle}\left(\frac{1-\hat{\theta}}{1-\theta}\right)^{\left\langle X_{t}, \bar{Y}_{t}\right\rangle} \mid \mathscr{Y}_{S}\right] \\
& =\mathbf{E}\left[(\log \hat{\theta}-\log \theta) \sum_{t \in S}\left\langle X_{t}, Y_{t}\right\rangle+(\log (1-\hat{\theta})-\log (1-\theta)) \sum_{t \in S}\left\langle X_{t}, \bar{Y}_{t}\right\rangle \mid \mathscr{Y}_{S}\right] \\
& =\log \hat{\theta} \mathbf{E}\left[\sum_{t \in S}\left\langle X_{t}, Y_{t}\right\rangle \mid \mathscr{Y}_{S}\right]+\log (1-\hat{\theta}) \mathbf{E}\left[\sum_{t \in S}\left\langle X_{t}, \bar{Y}_{t}\right\rangle \mid \mathscr{Y}_{S}\right]+R \\
& =\log \hat{\theta} E_{1}+\log (1-\hat{\theta}) E_{2}+R,
\end{aligned}
$$


где $E_{1}, E_{2}$ и $R$ не зависят от $\hat{\theta}$. Найдем максимум функции

$$
f(\hat{\theta})=\log \hat{\theta} E_{1}+\log (1-\hat{\theta}) E_{2}+R
$$

при $0<\hat{\theta}<1$. Из равенства

$$
f^{\prime}(\hat{\theta})=\frac{1}{\hat{\theta}} E_{1}-\frac{1}{1-\hat{\theta}} E_{2}=0
$$

следует, что $\hat{\theta}=E_{1} /\left(E_{1}+E_{2}\right)$. Значение $\hat{\theta}$, максимизирующее $\mathbf{E}\left[\log \Psi_{S} \mid \mathscr{Y}_{S}\right]$, определяется равенством

$$
\begin{aligned}
\hat{\theta} & =\frac{\mathbf{E}\left[\sum_{t \in S}\left\langle X_{t}, Y_{t}\right\rangle \mid \mathscr{Y}_{S}\right]}{\mathbf{E}\left[\sum_{t \in S}\left\langle X_{t}, Y_{t}\right\rangle \mid \mathscr{Y}_{S}\right]+\mathbf{E}\left[\sum_{t \in S}\left\langle X_{t}, \bar{Y}_{t}\right\rangle \mid \mathscr{Y}_{S}\right]} \\
& =\frac{1}{|S|} \sum_{t \in S} \mathbf{E}\left[\left\langle X_{t}, Y_{t}\right\rangle \mathscr{Y}_{S}\right]=\frac{\sum_{t \in S} q_{t}}{\sum_{t \in S}\left(q_{t}+q_{\bar{t}}\right)} .
\end{aligned}
$$

Теорема доказана.

Рассмотрим набор параметров СММ

$$
A=\left\{a^{e_{n}}\left(e_{k_{1}}, e_{k_{2}}, e_{k_{3}}, e_{k_{4}}\right), 1 \leqslant n, k_{i} \leqslant N, i=1,2,3,4\right\} .
$$

Требуется найти новый набор параметров

$$
\hat{A}=\left\{\widehat{a}^{e_{n}}\left(e_{k_{1}}, e_{k_{2}}, e_{k_{3}}, e_{k_{4}}\right), 1 \leqslant n, k_{i} \leqslant N, i=1,2,3,4\right\},
$$

который бы максимизировал условную функцию правдоподобия, описанную выше. Положим

$$
\Phi_{S}=\prod_{t \in S} \prod_{z_{t} \in \mathbf{X}_{t}} \prod_{z_{\delta(t)} \in \mathbf{X}_{\delta(t)}}\left(\frac{\hat{a}^{z_{t}}\left(z_{\delta(t)}\right)}{a^{z_{t}}\left(z_{\delta(t)}\right)}\right)^{\left\langle X_{t}, z_{t}\right\rangle \prod_{s \in \delta(t)}\left(X_{s}, z_{s}\right\rangle} .
$$

Тогда

$$
\begin{aligned}
& \mathbf{E}\left[\log \Phi_{S} \mid \mathscr{Y}_{S}\right] \\
& =\mathbf{E}\left[\sum_{t \in S} \sum_{z_{t} \in \mathbf{X}_{t}} \sum_{z_{\delta(t)} \in \mathbf{X}_{\delta(t)}}\left\langle X_{t}, z_{t}\right\rangle \prod_{s \in \delta(t)}\left\langle X_{S}, z_{s}\right\rangle\left(\log \hat{a}^{z_{t}}\left(z_{\delta(t)}\right)-\log a^{z_{t}}\left(z_{\delta(t)}\right)\right) \mid \mathscr{Y}_{S}\right] \\
& =\sum_{t \in S} \sum_{z_{t} \in \mathbf{X}_{t} \mid Y_{t}} \sum_{z_{\delta(t)} \in \mathbf{X}_{\delta(t)} \mid Y_{\delta(t)}} \mathbf{E}\left[\left\langle X_{t}, z_{t}\right\rangle \prod_{s \in \delta(t)}\left\langle X_{s}, z_{S}\right\rangle \mid \mathscr{Y}_{S}\right] \log \hat{a}^{z_{t}}\left(z_{\delta(t)}\right)+R^{\prime},
\end{aligned}
$$

где $R^{\prime}$ не зависит от $\hat{A}$. С учетом ограничений на элементы $\hat{A}$, которые состоят в том, что для всех $z_{\delta(t)} \in \mathbf{X}_{\delta(t)}$

$$
\sum_{z_{t} \in \mathbf{X}_{t}} \hat{a}^{z_{t}}\left(z_{\delta(t)}\right)=1
$$

и которые можно представить в динамической форме

$$
\sum_{t \in S} \sum_{z_{t} \in \mathbf{X}_{t}} \sum_{z_{\delta(t)} \in \mathbf{X}_{\delta(t)}} \hat{a}^{z_{t}}\left(z_{\delta(t)}\right) \prod_{s \in \delta(t)}\left\langle X_{S}, z_{s}\right\rangle=\sum_{t \in S} \sum_{z_{t} \in \mathbf{X}_{t}} \hat{a}^{z_{t}}\left(X_{\delta(t)}\right)=|S|,
$$


а также в условной форме

$$
\begin{aligned}
\mathbf{E}\left[\sum_{t \in S} \sum_{z_{t} \in \mathbf{X}_{t}} \sum_{z_{\delta(t)} \in \mathbf{X}_{\delta(t)}} \hat{a}^{z_{t}}\left(z_{\delta(t)}\right) \prod_{s \in \delta(t)}\left\langle X_{s}, z_{s}\right\rangle \mid \mathscr{Y}_{S}\right] \\
=\sum_{t \in S} \sum_{z_{t} \in \mathbf{X}_{t}} \sum_{z_{\delta(t)} \in \mathbf{X}_{\delta(t)} \mid Y_{\delta(t)}} \hat{a}^{z_{t}}\left(z_{\delta(t)}\right) \mathbf{E}\left[\prod_{s \in \delta(t)}\left\langle X_{S}, z_{S}\right\rangle \mathscr{Y}_{S}\right]=|S|,
\end{aligned}
$$

параметры, максимизирующие $E\left[\log \Phi_{S} \mid \mathscr{Y}_{S}\right]$, можно найти, используя метод множителей Лагранжа.

Рассмотрим функцию

$$
\begin{aligned}
g(\hat{A}, \lambda)= & \sum_{t \in S} \sum_{z_{t} \in \mathbf{X}_{t} \mid Y_{t}} \sum_{z_{\delta(t)} \in \mathbf{X}_{\delta(t)} \mid Y_{\delta(t)}} \mathbf{E}\left[\left\langle X_{t}, z_{t}\right\rangle \prod_{s \in \delta(t)}\left\langle X_{S}, z_{S}\right\rangle \mid \mathscr{Y}_{S}\right] \log \hat{a}^{z_{t}}\left(z_{\delta(t)}\right) \\
& +R^{\prime}+\lambda\left(\sum_{t \in S} \sum_{z_{t} \in \mathbf{X}_{t}} \sum_{z_{\delta(t)} \in \mathbf{X}_{\delta(t)} \mid Y_{\delta(t)}} \hat{a}^{z_{t}}\left(z_{\delta(t)}\right) \mathbf{E}\left[\prod_{S \in \delta(t)}\left\langle X_{S}, z_{S}\right\rangle \mid \mathscr{Y}_{S}\right]-|S|\right) .
\end{aligned}
$$

Уравнение Лагранжа имеет вид

$$
\begin{aligned}
\frac{\partial g}{\partial \hat{a}^{e_{n}}\left(e_{k_{1}}, e_{k_{2}}, e_{k_{3}}, e_{k_{4}}\right)}=\frac{\sum_{1} \mathrm{E}\left[I\left(X \overline{\delta(t)} ; e_{n}, e_{k_{1}}, e_{k_{2}}, e_{k_{3}}, e_{k_{4}}\right) \mid \mathscr{Y}_{S}\right]}{\hat{a}^{e_{n}}\left(e_{k_{1}}, e_{k_{2}}, e_{k_{3}}, e_{k_{4}}\right)} \\
+\lambda \sum_{2} \mathrm{E}\left[I\left(X_{\delta(t)} ; e_{k_{1}}, e_{k_{2}}, e_{k_{3}}, e_{k_{4}}\right) \mid \mathscr{Y}_{S}\right]=0
\end{aligned}
$$

где символ $\sum_{1}$ есть знак суммирования по $t \in S:\left\{e_{n}, e_{k_{1}}, e_{k_{2}}, e_{k_{3}}, e_{k_{4}}\right\} \in \mathbf{X}_{\overline{\delta(t)}} \mid Y_{\overline{\delta(t)}}$, символ $\sum_{2}$ есть знак суммирования по $t \in S:\left\{e_{k_{1}}, e_{k_{2}}, e_{k_{3}}, e_{k_{4}}\right\} \in \mathbf{X}_{\delta(t)} \mid Y_{\delta(t)}$,

$$
\begin{array}{rlrl}
I\left(X_{\delta(t)} ; e_{k_{1}}, e_{k_{2}}, e_{k_{3}}, e_{k_{4}}\right) & =\prod_{1 \leqslant i \leqslant 4}\left\langle X_{s_{i}}, e_{k_{i}}\right\rangle, & \left\{s_{1}, s_{2}, s_{3}, s_{4}\right\}=\delta(t), \\
I\left(X_{\overline{\delta(t)}} ; e_{n}, e_{k_{1}}, e_{k_{2}}, e_{k_{3}}, e_{k_{4}}\right) & =\left\langle X_{t}, e_{n}\right\rangle I\left(X_{\delta(t)} ; e_{k_{1}}, e_{k_{2}}, e_{k_{3}}, e_{k_{4}}\right), & 1 \leqslant n, & k_{i} \leqslant N .
\end{array}
$$

Тогда

$$
\widehat{a}^{e_{n}}\left(e_{k_{1}}, e_{k_{2}}, e_{k_{3}}, e_{k_{4}}\right)=-\frac{\sum_{1} \mathrm{E}\left[I\left(X_{\overline{\delta(t)}} ; e_{n}, e_{k_{1}}, e_{k_{2}}, e_{k_{3}}, e_{k_{4}}\right) \mid \mathscr{Y}_{S}\right]}{\lambda \sum_{2} \mathrm{E}\left[I\left(X_{\delta(t)} ; e_{k_{1}}, e_{k_{2}}, e_{k_{3}}, e_{k_{4}}\right) \mid \mathscr{Y}_{S}\right]},
$$

учитывая (4) и то, что

$$
\begin{aligned}
\sum_{n=1}^{N} \sum_{1} \mathbf{E}\left[I\left(X \overline{\delta(t)} ; e_{n}, e_{k_{1}}, e_{k_{2}}, e_{k_{3}}, e_{k_{4}}\right) \mid \mathscr{Y}_{S}\right] \\
=\sum_{2} \mathbf{E}\left[\left(\sum_{n=1}^{N}\left\langle X_{t}, e_{n}\right\rangle\right) I\left(X_{\delta(t)} ; e_{k_{1}}, e_{k_{2}}, e_{k_{3}}, e_{k_{4}}\right) \mid \mathscr{Y}_{S}\right] \\
=\sum_{2} \mathbf{E}\left[I\left(X_{\delta(t)} ; e_{k_{1}}, e_{k_{2}}, e_{k_{3}}, e_{k_{4}}\right) \mid \mathscr{Y}_{S}\right]
\end{aligned}
$$


получаем, что $\lambda=-1$ и, следовательно,

$$
\begin{aligned}
\hat{a}^{e_{n}}\left(e_{k_{1}}, e_{k_{2}}, e_{k_{3}}, e_{k_{4}}\right)= & \frac{\sum_{1} \mathbf{E}\left[I\left(X_{\overline{\delta(t)}} ; e_{n}, e_{k_{1}}, e_{k_{2}}, e_{k_{3}}, e_{k_{4}}\right) \mid \mathscr{Y}_{S}\right]}{\sum_{2} \mathbf{E}\left[I\left(X_{\delta(t)} ; e_{k_{1}}, e_{k_{2}}, e_{k_{3}}, e_{k_{4}}\right) \mid \mathscr{Y}_{S}\right]} \\
= & \frac{\sum_{1} q_{\overline{\delta(t)}}\left(e_{n}, e_{k_{1}}, e_{k_{2}}, e_{k_{3}}, e_{k_{4}}\right)}{\sum_{2} q_{\delta(t)}\left(e_{k_{1}}, e_{k_{2}}, e_{k_{3}}, e_{k_{4}}\right)},
\end{aligned}
$$

где

$$
\begin{aligned}
q_{\delta(t)}\left(e_{k_{1}}, e_{k_{2}}, e_{k_{3}}, e_{k_{4}}\right)= & \hat{\mathbf{E}}\left[I\left(X_{\delta(t)} ; e_{k_{1}}, e_{k_{2}}, e_{k_{3}}, e_{k_{4}}\right) \bar{\Lambda}_{S} \bar{\Gamma}_{S} \mid \mathscr{Y}_{S}\right] \\
= & \left(\frac{2}{N}\right)^{4} \theta^{\left|\left\{i: e_{k_{i}}=Y_{s_{i}}\right\}\right|}(1-\theta)^{\left|\left\{i: e_{k_{i}}=\bar{Y}_{s_{i}}\right\}\right|} \\
& \times \hat{\mathbf{E}}\left[\bar{\Lambda}_{S \backslash \delta(t)} \bar{\Gamma}_{S} \mid X_{s_{i}}=e_{k_{i}}, 1 \leqslant i \leqslant 4, \Psi_{S}\right] \\
= & \left(\frac{2}{N}\right)^{4} \theta^{\left|\left\{i: e_{k_{i}}=Y_{s_{i}}\right\}\right|}(1-\theta)^{\left|\left\{i: e_{k_{i}}=\bar{Y}_{s_{i}}\right\}\right|} \beta_{S \backslash \delta(t)}\left(e_{k_{1}}, e_{k_{2}}, e_{k_{3}}, e_{k_{4}}\right), \\
q_{\overline{\delta(t)}}\left(e_{n}, e_{k_{1}}, e_{k_{2}}, e_{k_{3}}, e_{k_{4}}\right)= & \hat{\mathbf{E}}\left[I\left(X_{\overline{\delta(t)}} ; e_{n}, e_{k_{1}}, e_{k_{2}}, e_{k_{3}}, e_{k_{4}}\right) \bar{\Lambda}_{S} \bar{\Gamma}_{S} \mid \mathscr{Y}_{S}\right] \\
= & \left(\frac{2}{N}\right)^{5} \theta^{\left|\left\{i: e_{k_{i}}=Y_{s_{i}}\right\}\right|+\left(e_{n}, Y_{t}\right\rangle}(1-\theta)^{\left|\left\{i: e_{k_{i}}=\bar{Y}_{s_{i}}\right\}\right|+\left\langle e_{n}, \bar{Y}_{t}\right\rangle} \\
& \times \beta_{S \backslash \overline{\delta(t)}}\left(e_{n}, e_{k_{1}}, e_{k_{2}}, e_{k_{3}}, e_{k_{4}}\right) .
\end{aligned}
$$

Таким образом, справедлива следующая теорема.

Теорема 8. Оченка условного максимального правдоподобия для набора параметров СММ А при известных значениях $Y_{t}, t \in S$, на $M$-шаге определяется по формуле

$$
\hat{a}^{e_{n}}\left(e_{k_{1}}, e_{k_{2}}, e_{k_{3}}, e_{k_{4}}\right)=\frac{\sum_{1} q_{\delta(t)}\left(e_{n}, e_{k_{1}}, e_{k_{2}}, e_{k_{3}}, e_{k_{4}}\right)}{\sum_{2} q_{\delta(t)}\left(e_{k_{1}}, e_{k_{2}}, e_{k_{3}}, e_{k_{4}}\right)}
$$

где $\sum_{1}, \sum_{2}, q_{\overline{\delta(t)}}\left(e_{n}, e_{k_{1}}, e_{k_{2}}, e_{k_{3}}, e_{k_{4}}\right) u q_{\delta(t)}\left(e_{k_{1}}, e_{k_{2}}, e_{k_{3}}, e_{k_{4}}\right)$ определялись выше.

Аналогичные теоремы можно сформулировать и для условных вероятностей для граничных пикселей, то есть для тех $t \in S$, для которых $|\delta(t)|<4$.

Две последние теоремы завершают построение ЕМ-алгоритма для оценки параметров скрытого марковского поля в случае LSB-модификации исходного дискретного марковского поля.

\section{4. Заключение}

В данной работе был рассмотрен подход к решению задач стегоанализа с использованием скрытых марковских моделей. Решена задача оценки параметров скрытого марковского поля при LSB-модификации исходного дискретного марковского поля, моделирующего прямоугольное растровое изображение. Для решения этой задачи использован известный итерационный ЕМ-алгоритм максимизации условного отношения правдоподобия. Подход, предложенный в работе, может быть использован как для стегоанализа изображений, модифицированных с помощью более сложных стеганографических методов (встраивающих информацию не только в незначащие биты), так и для других задач обработки изображений и сигналов. 


\section{Список литературы}

1. Winkler G., Image analysis, random fields and dynamic Monte Carlo methods. Springer, Berlin, 1995.

2. Elliott R. J., Aggoun L., Moore J. B., Hidden Markov models: estimation and control. Springer, Berlin, 1995.

3. Рабинер Л. Р., Скрытые марковские модели и их применение в избранных приложениях при распознавании речи. ТИИЭР (1989) 77, №2, 86-120.

4. Сидоров М. А., Скрытые марковские модели и стегоанализ. В сб.: Материалы VI Международной научно-практической конференции «Информачионная безопасность». ТРТУ, Таганрог, 2004, c. 464.

5. Sidorov M., Hidden Markov models and steganalysis. In: Proc. ACM Workshop on Multimedia and Security, 2004. ACM Press, New York, 2004, 63-67.

6. Johnson N. F., Duric Z., Jajodia S., Information hiding: steganography and watermarking-attacks and countermeasures. Kluwer, New York, 2000.

7. Petitcolas F. A. P., Anderson R. J., Kuhn M. G., Information hiding-a survey. Proc. IEEE (1999) 87, 1062-1078.

8. Fridrich J., Goljan M, Du R., Reliable detection of LSB steganography in grayscale and color images. In: Proc. ACM Workshop on Multimedia and Security, 2001. ACM Press, New York, 2001, pp. 2730.

9. Булинский А. В., Ширяев А. Н., Теория случайных прочессов. Физматлит, Москва, 2003.

10. Халмош П., Теория меры. Факториал, Москва, 2003.

11. Baum L.E., Petrie T., Soules G., Weiss N., A maximization technique occuring in the statistical analysis of probabilistic functions of Markov chains. Ann. Math. Stat. (1970) 41, 164-171.

Статья поступила 20.10.2004. 